\title{
JACOBI POLYNOMIALS AS GENERALIZED FABER POLYNOMIALS
}

\author{
AHMED I. ZAYED
}

\begin{abstract}
Let $\mathbf{B}$ be an open bounded subset of the complex $z$-plane with closure $\overline{\mathbf{B}}$ whose complement $\overline{\mathbf{B}}^{c}$ is a simply connected domain on the Riemann sphere. Let $z=\psi(w)$ map the domain $|w|>\rho \quad(\rho>0)$ one-to-one conformally onto the domain $\overline{\mathbf{B}}^{c}$ such that $\psi(\infty)=\infty$. Let $R(w)=\sum_{n=0}^{\infty} c_{n} w^{-n}$, $c_{0} \neq 0$ be analytic in the domain $|w|>\rho$ with $R(w) \neq 0$. Let $F(z)=$ $\sum_{n=0}^{\infty} b_{n} z^{n}, b_{n} \neq 0, F *(z)=\sum_{n=0}^{\infty} \frac{1}{b_{n}} z^{n}$ be analytic in $|z|<1$ and analytically continuable to any point outside $|z|<1$ along any path not passing through the points $z=0,1, \infty$.

The generalized Faber polynomials $\left\{P_{n}(z)\right\}_{n=0}^{\infty}$ of $\mathbf{B}$ are defined by$$
\frac{t \psi^{\prime}(t)}{\psi(t)} R(t) F\left(\frac{z}{\psi(t)}\right)=\sum_{n=0}^{\infty} P_{n}(z) \frac{1}{t^{n}}, \quad|t|>\rho .
$$

The aim of this paper is to show that

(1) if the Jacobi polynomials $\left\{P_{n}^{(\alpha, \beta)}(z)\right\}_{n=0}^{\infty}$ are generalized Faber polynomials of any region $\mathbf{B}$, then it must be the elliptic region $\{z:|z+1|+|z-1|<$ $\left.\rho+\frac{1}{\rho}, \rho>1\right\}$

(2) the only Jacobi polynomials that can be classified as generalized Faber polynomials are the Tchebycheff polynomials of the first kind, some normalized Gegenbauer polynomials, some normalized Jacobi polynomials of type $\left\{P_{n}^{(\alpha, \alpha+1)}(z)\right\}_{n=0}^{\infty}, \quad\left\{P_{n}^{(\beta+1, \beta)}(z)\right\}_{n=0}^{\infty}$ and there are no others, no matter how one normalizes them;

(3) the Hermite and Laguerre polynomials cannot be generalized Faber polynomials of any region.

\section{INTRODUCTION}

Faber polynomials, which were introduced by Faber in 1903 [2] and later developed by several Russian mathematicians (see [7 and 9] for references), play an important role in the theory of functions of a complex variable and in approximation theory.

One of their most important properties is that the Faber polynomials $\left\{P_{n}(z)\right\}_{n=0}^{\infty}$ of a domain $\mathbf{B}$ play in $\mathbf{B}$ the role that $\left\{z^{n}\right\}_{n=0}^{\infty}$ play in the unit disc, i.e., any analytic function $f(z)$ in $\mathbf{B}$ can be expanded in a series of Faber

Received by the editors October 30, 1987 and, in revised form, November 7, 1988. Presented at the 94th annual meeting of the AMS in Atlanta, Georgia, January 1988.

1980 Mathematics Subject Classification (1985 Revision). Primary 33A65; Secondary 30C10.

Key words and phrases. Jacobi polynomials, Faber polynomials. 
polynomials $f(z)=\sum_{n=0}^{\infty} a_{n} P_{n}(z)$, where the series converges uniformly to $f(z)$ on compact subsets of $\mathbf{B}$.

Although not necessarily orthogonal, they have been used in the theory of orthogonal polynomials and special functions by several people including $Y$. Geronimus [3], V. Smirnov and N. Lebedev [7], P. Suetin [10], G. Szegö [12] in connection with polynomials orthogonal on intervals, curves and by $\mathrm{P}$. Suetin [11] in connection with polynomials orthogonal on domains.

To introduce the Faber polynomials, let us assume that $\mathbf{B}$ is an open bounded subset of the complex $z$-plane with closure $\overline{\mathbf{B}}$ whose complement $\overline{\mathbf{B}}^{c}$ is a simply connected domain on the Riemann sphere $\mathbf{Q}$. Let $z=\psi(w)$ map the domain $|w|>\rho \quad(\rho>0)$ one-to-one conformally onto the domain $\overline{\mathbf{B}}^{c}$ such that $\psi(\infty)=\infty$. We denote the inverse function of $z=\psi(w)$ by $w=\phi(z)$. Let $L_{r}$ be the image of $\partial D_{r}=\{w:|w|=r\}$, when mapped by the function $z=\psi(w)$ and $\mathbf{B}_{r}$ be the bounded domain with boundary $L_{r}$. The boundary of $\mathbf{B}$ will be denoted by $L$. Let

$$
R(w)=\sum_{n=0}^{\infty} c_{n} w^{-n}, \quad c_{0} \neq 0,
$$

be analytic in the domain $|w|>\rho$ with $R(w) \neq 0$ therein. The Faber polynomials $\left\{P_{n}(z)\right\}_{n=0}^{\infty}$ of the domain $\mathbf{B}$ are defined by

$$
\frac{t \psi^{\prime}(t)}{\psi(t)-z} R(t)=\sum_{n=0}^{\infty} P_{n}(z) \frac{1}{t^{n}}, \quad|t|>\rho .
$$

It can be shown [7] that, when the generating function $\frac{t \psi^{\prime}(t)}{\psi(t)-z} R(t)$ is expanded as a Laurent series in some neighbourhood of $t=\infty, P_{n}(z)$ is indeed a polynomial in $z$ of exact degree $n$.

One of the aims of this paper is to study the relationship between Faber polynomials and some of the classical orthogonal polynomials. Unfortunately, the class of Faber polynomials defined by (1.2) is so limited that the only classical system of orthogonal polynomials it contains is the Tchebycheff polynomials of the first kind. A larger class of Faber polynomials, known as the generalized Faber polynomials, was introduced by V. Smirnov and N. Lebedev [7] as follows:

Let

$$
F(z)=\sum_{n=0}^{\infty} b_{n} z^{n}, \quad b_{n} \neq 0,
$$

be analytic in $|z|<1$ and assume that $F(z)$ can be continued analytically to any point outside the unit disc by any path not passing through the points $z=0,1, \infty$. If the same is true for the function

$$
F_{*}(z)=\sum_{n=0}^{\infty} \frac{1}{b_{n}} z^{n},
$$


we say that $F(z)$ and $F_{*}(z)$ are adjoint. In this case it is easy to see that the point $z=1$ is a singular point for both $F(z)$ and $F_{*}(z)$.

Let the $\mathbf{B}$ be as before. Furthermore, let us assume that the point $z=0$ belongs to $\mathbf{B}$ and that $F(z), F_{*}(z)$ are adjoint. Then, the generalized Faber polynomials $\left\{P_{n}(z)\right\}_{n=0}^{\infty}$ of $\mathbf{B}$ are defined by

$$
\frac{t \psi^{\prime}(t)}{\psi(t)} R(t) F\left(\frac{z}{\psi(t)}\right)=\sum_{n=0}^{\infty} P_{n}(z) \frac{1}{t^{n}}, \quad|t|>\rho .
$$

Again, it can be shown that $P_{n}(z)$ is a polynomial in $z$ of exact degree $n$. Moreover, if $f(z)$ is analytic in the domain $\mathbf{B}_{r}$ and has a singular point on $L_{r}$, then

$$
f(z)=\sum_{n=0}^{\infty} a_{n} P_{n}(z), \quad z \in \mathbf{B}_{r}
$$

where

$$
a_{n}=\frac{1}{2 \pi i} \int_{L_{r_{1}}} f(\xi) q_{n}(\xi) d \xi, \quad \rho<r_{1}<r
$$

and

$$
\varlimsup_{n \rightarrow \infty} \sqrt[n]{\left|a_{n}\right|}=\frac{1}{r}, \quad \rho<r<\infty
$$

and

$$
q_{n}(\xi)=\frac{1}{2 \pi i} \int_{|t|=r_{1}} F_{*}\left(\frac{\psi(t)}{\xi}\right) \frac{1}{\xi R(t)} \frac{1}{t^{n+1}} d t, \quad \xi \in \overline{\mathbf{B}}_{r}^{c} .
$$

Clearly, (1.2) is a special case of (1.5) when $F(u)=\frac{1}{(1-u)}$.

In a recent paper [13], the authors showed that an analytic function $f(z)$ in B given by (1.6) has a singular point at $z=z_{1}$ if and only if

$$
g(w)=\sum_{n=0}^{\infty} a_{n} w^{n}
$$

has one at $w=w_{1}$, where $w_{1}=\phi\left(z_{1}\right)$, i.e., $z_{1}=\psi\left(w_{1}\right)$ and deduced an old result by $R$. Gilbert [5] that a series of Gegenbauer polynomials

$$
f(z)=\sum_{n=0}^{\infty} a_{n} C_{n}^{\lambda}(z), \quad \varlimsup_{n \rightarrow \infty} \sqrt[n]{\left|a_{n}\right|}=\frac{1}{\rho}, \quad \rho>1, \lambda>-\frac{1}{2}, \lambda \neq 0,
$$

has a singular point $z=z_{1}$ if and only if $g(w)$ has one at $w=w_{1}$ where

$$
z_{1}=\frac{1}{2}\left(w_{1}+\frac{1}{w_{1}}\right) \text {. }
$$

A key point in their proof is to show that the Gegenbauer polynomials are generalized Faber polynomials of the elliptic region

$$
\mathscr{A}=\left\{z:|z+1|+|z-1|<\rho+\frac{1}{\rho}\right\} .
$$


A proof of this fact will be given in the following section. It should be mentioned that Gilbert's result is, in fact, a generalization of an earlier result by $Z$. Nehari [6] who showed that a series of Legendre polynomials

$$
f(z)=\sum_{n=0}^{\infty} a_{n} P_{n}(z), \varlimsup_{n \rightarrow \infty} \sqrt[n]{\left|a_{n}\right|}=\frac{1}{\rho}, \quad \rho>1,
$$

has a singular point at $z=z_{1}$ if and only if $g(w)$ has one at $w=w_{1}$ where $z_{1}$ and $w_{1}$ are related as in (1.12). For $\lambda=\frac{1}{2}$, Gilbert's result yields Nehari's.

Although a similar result concerning the location of the singularities of series of Jacobi polynomials

$$
\sum_{n=0}^{\infty} a_{n} P_{n}^{(\alpha, \beta)}(z), \quad \alpha, \beta>-1,
$$

was proved by R. Gilbert [4], the authors in [13] were unable to deduce it from their general theorem concerning the location of the singularities of series of generalized Faber polynomials since it was not known whether the Jacobi polynomials were generalized Faber polynomials of any region $\mathbf{B}$. It was also conjectured that the Jacobi polynomials might be generalized Faber polynomials of some domain $\mathbf{B}^{(\alpha, \beta)}$ that depends on $\alpha, \beta$ and which reduces to the elliptic region $\mathscr{A}$ when $\alpha=\beta$.

The aim of this paper is to show that:

(1) the above conjecture is false, i.e., if the Jacobi polynomials are generalized Faber polynomials of any region, then it must essentially be the elliptic region $\mathscr{A}$.

(2) the only Jacobi polynomials that can be classified as generalized Faber polynomials are the Tchebycheff polynomials of the first kind, some normalized Gegenbauer polynomials, some normalized Jacobi polynomials of type

$$
\left\{P_{n}^{(\alpha, \alpha+1)}(z)\right\}_{n=0}^{\infty}, \quad\left\{P_{n}^{(\beta+1, \beta)}(z)\right\}_{n=0}^{\infty}
$$

and there are no others, no matter how one normalizes them.

(3) the Hermite and Laguerre polynomials cannot be generalized Faber polynomials of any region.

\section{Preliminaries}

For any complex number $a$ and nonnegative integer $n$, let

$$
\begin{aligned}
(a)_{0} & =1, \quad(a)_{n}=a(a+1) \cdots(a+n-1)=\frac{\Gamma(a+n)}{\Gamma(a)}, \\
\left(\begin{array}{l}
a \\
n
\end{array}\right) & =\frac{\Gamma(a+1)}{\Gamma(n+1) \Gamma(a-n+1)} .
\end{aligned}
$$


For any complex numbers $a, b$ and $c$ with $c \neq 0,-1,-2, \ldots$, the hypergeometric function $F(a, b ; c ; z)={ }_{2} F_{1}(a, b ; c ; z)$ is defined by

$$
F(a, b ; c ; z)=\sum_{n=0}^{\infty} \frac{(a)_{n}(b)_{n}}{(c)_{n} n !} z^{n}, \quad|z|<1 .
$$

The Jacobi polynomials $P_{n}^{(\alpha, \beta)}(x), n=0,1,2, \ldots, \alpha, \beta>-1$, are defined by

$$
P_{n}^{(\alpha, \beta)}(x)=\left(\begin{array}{c}
n+\alpha \\
n
\end{array}\right) F\left(-n, n+\alpha+\beta+1 ; \alpha+1 ; \frac{1-x}{2}\right)
$$

hence

$$
P_{n}^{(\alpha, \beta)}(1)=\left(\begin{array}{c}
n+\alpha \\
n
\end{array}\right)=\frac{(\alpha+1)_{n}}{n !}
$$

$P_{n}^{(\alpha, \beta)}(x)$ is a polynomial solution of the differential equation

$$
\left(1-x^{2}\right) y^{\prime \prime}+[(\beta-\alpha)-(\alpha+\beta+2) x] y^{\prime}+n(n+\alpha+\beta+1) y=0 .
$$

They form a complete orthogonal system in $L^{2}\left((-1,1), d W^{(\alpha, \beta)}(x)\right\}$ where

$$
d W^{(\alpha, \beta)}(x)=(1-x)^{\alpha}(1+x)^{\beta} d x .
$$

The orthogonality relation reads

$$
\int_{-1}^{1} P_{n}^{(\alpha, \beta)}(x) P_{m}^{(\alpha, \beta)}(x)(1-x)^{\alpha}(1+x)^{\beta} d x=h_{n} \delta_{n m}
$$

where

$$
h_{n}=\frac{2^{\alpha+\beta+1} \Gamma(n+\alpha+1) \Gamma(n+\beta+1)}{(2 n+\alpha+\beta+1) \Gamma(n+1) \Gamma(n+\alpha+\beta+1)} .
$$

The following special cases of the Jacobi polynomials are of some importance to us:

(1) The Gegenbauer polynomials $C_{n}^{\lambda}(x)$, also known as the ultraspherical polynomials, are defined by

$$
C_{n}^{\lambda}(x)=\frac{\Gamma\left(\lambda+\frac{1}{2}\right) \Gamma(n+2 \lambda)}{\Gamma(2 \lambda) \Gamma\left(n+\lambda+\frac{1}{2}\right)} P_{n}^{(\lambda-1 / 2, \lambda-1 / 2)}(x), \quad \lambda>-\frac{1}{2} .
$$

(2) The Legendre polynomials $P_{n}(x)$ are $P_{n}(x)=C_{n}^{1 / 2}(x)=P_{n}^{(0,0)}(x)$.

(3) The Tchebycheff polynomials of the first and second kinds are given respectively by

$$
T_{n}(x)=\frac{P_{n}^{(-1 / 2,-1 / 2)}(x)}{P_{n}^{(-1 / 2,-1 / 2)}(1)}=\cos n \theta, \quad x=\cos \theta,
$$


and

$$
U_{n}(x)=C_{n}^{1}(x) .
$$

Finally, let $\rho>1$ and $c$ be a nonzero real number. Then, the exterior of the circle $|t|=\rho$ is mapped one-to-one under the conformal mapping

$$
z=\frac{1}{2}\left(c t+\frac{1}{c t}\right)
$$

onto the exterior of the ellipse $\partial \mathscr{A}(c)$ whose foci are at \pm 1 with semi-axis

$$
\frac{1}{2}\left|\left(c \rho+\frac{1}{c \rho}\right)\right| \text { and } \frac{1}{2}\left|\left(c \rho-\frac{1}{c \rho}\right)\right|
$$

provided that we take the branch of the inverse map $t=z+\sqrt{z^{2}-1}$ which becomes infinite at $z=\infty$. We denote the elliptic region bounded by $\partial \mathscr{A}(c)$ by $\mathscr{A}(c)$. In particular,

$$
\mathscr{A}=\mathscr{A}(1)=\left\{z:|z-1|+|z+1|<\rho+\frac{1}{\rho}\right\} .
$$

\section{THE MAIN RESULTS}

This section contains the main results which are formulated in three theorems and a corollary.

Theorem 1. The following sets of Jacobi polynomials are generalized Faber polynomials of the elliptic region $\mathscr{A}$.

(a) $\left\{T_{0}(x), 2 T_{n}(x)\right\}_{n=1}^{\infty}$,

(b) $\left\{C_{n}^{\lambda}(x)\right\}_{n=0}^{\infty}, \quad \lambda \neq 0$,

(c) $\left\{\frac{(n+\lambda)}{\lambda} C_{n}^{\lambda}(x)\right\}_{n=0}^{\infty}, \lambda \neq 0$,

(d) $\left\{\frac{(2 \alpha+2)_{n}}{(\alpha+1)_{n}} P_{n}^{(\alpha, \alpha+1)}(x)\right\}_{n=0}^{\infty}$,

(e) $\left\{\frac{(2 \beta+2)_{n}}{(\beta+1)_{n}} P_{n}^{(\beta+1, \beta)}(x)\right\}_{n=0}^{\infty}$.

Proof. (a) Since $T_{n}(\cos \theta)=\cos n \theta$, it follows from Poisson's sum

$$
1+2 \sum_{n=0}^{\infty}(\cos n \theta) r^{n}=\frac{1-r^{2}}{1-2 r \cos \theta+r^{2}}, \quad|r|<1
$$

that

$$
T_{0}(x)+\sum_{n=0}^{\infty} 2 T_{n}(x) \frac{1}{t^{n}}=\frac{\left(t^{2}-1\right)}{\left(t^{2}+1\right)}\left[1-\frac{x}{\frac{1}{2}(t+1 / t)}\right]^{-1}
$$

which is in the form given by (1.5) with $\psi(t)=\frac{1}{2}(t+1 / t)$,

$$
R(t)=1
$$

and 


$$
F(u)=\frac{1}{(1-u)} .
$$

(b) From the generating function $[1$, p. 21]

$$
\sum_{n=0}^{\infty} C_{n}^{\lambda}(x) \frac{1}{t^{n}}=\frac{t^{2 \lambda}}{\left(t^{2}-2 x t+1\right)^{\lambda}}, \quad|t|>1, \lambda \neq 0,
$$

one obtains

$$
\sum_{n=0}^{\infty} C_{n}^{\lambda}(x) \frac{1}{t^{n}}=\frac{t^{2 \lambda}}{\left(t^{2}+1\right)^{\lambda}}\left[1-\frac{x}{\frac{1}{2}(t+1 / t)}\right]^{-\lambda}
$$

which yields $(1.5)$ for $\psi(t)=\frac{1}{2}(t+1 / t)$

$$
R(t)=\frac{t^{2 \lambda}}{\left(t^{2}+1\right)^{\lambda-1}\left(t^{2}-1\right)}
$$

and

$$
F(u)=1 /(1-u)^{\lambda}
$$

(c) Similarly, from the generating function [1, p. 71]

$$
\sum_{n=0}^{\infty} \frac{(n+\lambda)}{\lambda} C_{n}^{\lambda}(x) \frac{1}{t^{n}}=\frac{t^{2 \lambda}\left(t^{2}-1\right)}{\left(t^{2}-2 x t+1\right)^{\lambda+1}}, \quad|t|>1, \lambda \neq 0,
$$

we obtain

$$
\sum_{n=0}^{\infty} \frac{(n+\lambda)}{\lambda} C_{n}^{\lambda}(x) \frac{1}{t^{n}}=\frac{t^{2 \lambda}\left(t^{2}-1\right)}{\left(t^{2}+1\right)^{\lambda+1}}\left[1-\frac{x}{\frac{1}{2}(t+1 / t)}\right]^{-(\lambda+1)}
$$

which yields $(1.5)$ for $\psi(t)=\frac{1}{2}(t+1 / t)$

$$
R(t)=t^{2 \lambda} /\left(t^{2}+1\right)^{\lambda}
$$

and

$$
F(u)=(1-u)^{-(\lambda+1)} .
$$

(d) Upon setting $\beta=\alpha+1$ and replacing $t$ by $1 / t$ in the generating function [1, p. 21]

$$
\begin{aligned}
& \sum_{n=0}^{\infty} \frac{(\alpha+\beta+1)_{n}}{(\alpha+1)_{n}} P_{n}^{(\alpha, \beta)}(x) t^{n} \\
& \quad=\frac{1}{(1-t)^{\alpha+\beta+1}} F\left(\frac{\alpha+\beta+1}{2}, \frac{\alpha+\beta+2}{2} ; \alpha+1 ; \frac{2 t(x-1)}{(1-t)^{2}}\right)
\end{aligned}
$$


we obtain

$$
\begin{gathered}
\sum_{n=0}^{\infty} \frac{(2 \alpha+2)_{n}}{(\alpha+1)_{n}} P_{n}^{(\alpha, \alpha+1)}(x) \frac{1}{t^{n}} \\
\quad=\frac{t^{2 \alpha+2}}{(t-1)^{2 \alpha+2}} F\left(\alpha+1, \alpha+\frac{3}{2} ; \alpha+1 ; \frac{2(x-1)}{(\sqrt{t}-1 / \sqrt{t})^{2}}\right) \\
=\frac{t^{2 \alpha+2}(t-1)}{\left(t^{2}+1\right)^{\alpha+3 / 2}}\left[1-\frac{x}{\frac{1}{2}(t+1 / t)}\right]^{-(\alpha+3 / 2)}
\end{gathered}
$$

since $F(a, b ; a ; z)=(1-z)^{-b}$.

Equation (3.11) yields (1.5) with $\psi(t)=\frac{1}{2}(t+1 / t)$

$$
\begin{aligned}
R(t) & =\frac{t^{2 \alpha+2}}{\left(t^{2}+1\right)^{\alpha+1 / 2}(t+1)} \quad \text { and } \\
F(u) & =(1-u)^{-(\alpha+3 / 2)} .
\end{aligned}
$$

(c) Upon using the generating function [8, p. 112]

$$
\begin{aligned}
& \sum_{n=0}^{\infty} \frac{(\alpha+\beta+1)_{n}}{(\beta+1)_{n}} P_{n}^{(\alpha, \beta}(x) r^{n} \\
& \quad=\frac{1}{(1+r)^{\alpha+\beta+1}} F\left(\frac{\alpha+\beta+1}{2}, \frac{\alpha+\beta+2}{2} ; \beta+1 ; \frac{2 r(x+1)}{(1+r)^{2}}\right)
\end{aligned}
$$

with $\alpha=\beta+1$ and $r=1 / t$ we obtain as in part (b)

$$
\begin{aligned}
\sum_{n=0}^{\infty} \frac{(2 \beta+2)_{n}}{(\beta+1)_{n}} P_{n}^{(\beta+1, \beta)}(x) \frac{1}{t^{n}} \\
\quad=\frac{t^{2 \beta+2}(t+1)}{\left(t^{2}+1\right)^{\beta+3 / 2}}\left[1-\frac{x}{\frac{1}{2}(t+1 / t)}\right]^{-(\beta+3 / 2)}
\end{aligned}
$$

which gives (1.5) with $\psi(t)=\frac{1}{2}(t+1 / t)$

$$
\begin{aligned}
R(t) & =\frac{t^{2 \beta+2}}{\left(t^{2}+1\right)^{\beta+1 / 2}(t-1)} \quad \text { and } \\
F(u) & =(1-u)^{-(\beta+3 / 2)} .
\end{aligned}
$$

Q.E.D.

It should be noted that although the polynomials $\left\{\frac{2}{(2 n-1)} T_{2 n-1}(x)\right\}_{n=1}^{\infty}$ have a generating function of the form (1.5), namely

$$
\sum_{n=1}^{\infty} \frac{2}{(2 n-1)} T_{2 n-1}(x) \frac{1}{t^{n}}=\ln \left\{\frac{1+2 x(t+1 / t)^{-1}}{1-2 x(t+1 / t)^{-1}}\right\}
$$


(see [12, p. 83]) with $\psi(t)=\frac{1}{2}(t+1 / t)$,

$$
R(t)=\frac{t^{2}+1}{t^{2}-1}
$$

and

$$
F(u)=\ln \left\{\frac{1+u}{1-u}\right\}
$$

they are not generalized Faber polynomials of the elliptic region $\mathscr{A}$ since they consist only of odd polynomials.

Theorem 2. Let $\left\{\varepsilon_{n}\right\}_{n=0}^{\infty}$ be any sequence of complex numbers such that the series $\sum_{n=0}^{\infty} \varepsilon_{n} P_{n}^{(\alpha, \beta)}(x) \frac{1}{t^{n}}$ converges for $x \in[-1,1]$ and $|t|>1$. Then, if the normalized Jacobi polynomials $\left\{\varepsilon_{n} P_{n}^{(\alpha, \beta)}(x)\right\}_{n=0}^{\infty}$ are generalized Faber polynomials of any region, then it must be the elliptic region $\mathscr{A}(c)$.

Proof. Assume that $\left\{\varepsilon_{n} P_{n}^{(\alpha, \beta)}(x)\right\}_{n=0}^{\infty}$ are generalized Faber polynomials of some domain B determined by the conformal mapping $z=\psi(t)$. Therefore, there exist $R(t)$ and $F(u)$ as described in $\S 1$ such that

$$
\sum_{n=0}^{\infty} \varepsilon_{n} P_{n}^{(\alpha, \beta)}(x) \frac{1}{t^{n}}=\frac{t \psi^{\prime}(t)}{\psi(t)} R(t) F\left(\frac{x}{\psi(t)}\right)=G(t) F\left(\frac{x}{\psi(t)}\right)
$$

where

$$
G(t)=\frac{t \psi^{\prime}(t)}{\psi(t)} R(t)
$$

Let

$$
2 q=\alpha+\beta+1
$$

and

$$
\mathscr{L}_{x}^{(\alpha, \beta)}=\left(1-x^{2}\right) \frac{d^{2}}{d x^{2}}+[(\beta-\alpha)-(\alpha+\beta+2) x] \frac{d}{d x} .
$$

From (2.6), we have

$$
\mathscr{L}_{x}^{(\alpha, \beta)} P_{n}^{(\alpha, \beta)}(x)=\left(q^{2}-(n+q)^{2}\right) P_{n}^{(\alpha, \beta)}(x)
$$

and thus by operating with $\mathscr{L}_{x}^{(\alpha, \beta)}$ on both sides of $(3.18)$, we obtain

or equivalently

$$
\begin{aligned}
\sum_{n=0}^{\infty} \varepsilon_{n}\left[q^{2}-(n+q)^{2}\right] P_{n}^{(\alpha, \beta)}(x) \frac{1}{t^{n}} & =\mathscr{L}_{x}^{(\alpha, \beta)} G(t) F\left(\frac{x}{\psi(t)}\right) \\
& =G(t) \mathscr{L}_{x}^{(\alpha, \beta)} F\left(\frac{x}{\psi(t)}\right)
\end{aligned}
$$

$$
\begin{aligned}
-\sum_{n=0}^{\infty} \varepsilon_{n}(n+q)^{2} P_{n}^{(\alpha, \beta)}(x) \frac{1}{t^{n}} \\
=G(t) \mathscr{L}_{x}^{(\alpha, \beta)} F\left(\frac{x}{\psi(t)}\right)-q^{2} G(t) F\left(\frac{x}{\psi(t)}\right) .
\end{aligned}
$$


From (2.6) and (3.18), it is easy to see that

$$
\sum_{n=0}^{\infty} \varepsilon_{n}(n+q)^{2} P_{n}^{(\alpha, \beta)}(x) \frac{1}{t^{n}}=t^{q+1} \frac{d}{d t} t \frac{d}{d t}\left(\frac{1}{t^{q}} G(t) F\left(\frac{x}{\psi(t)}\right)\right) .
$$

Thus, by combining (3.23) and (3.24) we obtain

$$
\begin{aligned}
G(t) \mathscr{L}_{x}^{(\alpha, \beta)} F\left(\frac{x}{\psi(t)}\right) & +t^{q+1} \frac{d}{d t} t \frac{d}{d t}\left(\frac{1}{t^{q}} G(t) F\left(\frac{x}{\psi(t)}\right)\right) \\
& -q^{2} G(t) F\left(\frac{x}{\psi(t)}\right)=0
\end{aligned}
$$

which, after somewhat involved computations, reduces to

$$
\begin{aligned}
\left(1-A(t) u^{2}\right) F^{\prime \prime}(u) & +[(\beta-\alpha) \psi(t)-B(t) \psi(t) u] F^{\prime}(u) \\
& +C(t) \psi^{2}(t) F(u)=0
\end{aligned}
$$

where

$$
\begin{aligned}
& u=\frac{x}{\psi(t)}, \\
& A(t)=\psi^{2}(t)-t^{2}\left[\psi^{\prime}(t)\right]^{2}, \\
& B(t)=(2 q+1) \psi(t)-(2 q-1) t \psi^{\prime}(t)+2 t^{2} \psi^{\prime}(t) \frac{G^{\prime}(t)}{G(t)} \\
& +t^{2} \psi^{\prime \prime}(t)-2 t^{2} \frac{\left[\psi^{\prime}(t)\right]^{2}}{\psi(t)}, \\
& C(t)=(1-2 q) t \frac{G^{\prime}(t)}{G(t)}+t^{2} \frac{G^{\prime \prime}(t)}{G(t)} \text {. }
\end{aligned}
$$

But since $G(t)=\frac{t \psi^{\prime}(t)}{\psi(t)} R(t)$, then

$$
\frac{G^{\prime}(t)}{G(t)}=\frac{1}{t}+\frac{\psi^{\prime \prime}(t)}{\psi^{\prime}(t)}+\frac{R^{\prime}(t)}{R(t)}-\frac{\psi^{\prime}(t)}{\psi(t)}
$$

and consequently

$$
\begin{aligned}
A(t)= & \left(\psi^{2}(t)-t^{2}\left[\psi^{\prime}(t)\right]^{2}\right), \\
B(t)= & (2 q+1) \psi(t)+(3-2 q) t \psi^{\prime}(t)+3 t^{2} \psi^{\prime \prime}(t) \\
& -4 t^{2} \frac{\left[\psi^{\prime}(t)\right]^{2}}{\psi(t)}+2 t^{2} \psi^{\prime}(t) \frac{R^{\prime}(t)}{R(t)}, \\
C(t)= & (1-2 q) t\left(\frac{G^{\prime}(t)}{G(t)}\right)+t^{2}\left(\frac{G^{\prime}(t)}{G(t)}\right)^{2}+t^{2} \frac{d}{d t}\left(\frac{G^{\prime}(t)}{G(t)}\right)
\end{aligned}
$$

where $\frac{G^{\prime}(t)}{G(t)}$ is given explicitly in terms of $R(t)$ by (3.27).

From (3.26), it follows that for $F(u)$ to have a singularity at $u=1, A(t)$ must be 1 . Therefore,

$$
\psi^{2}(t)-t^{2}\left[\psi^{\prime}(t)\right]^{2}=1
$$


which upon solving yields

$$
\psi(t)=\frac{1}{2}\left(c t+\frac{1}{c t}\right)
$$

and this completes the proof. Q.E.D.

In the next theorem we show that the only Jacobi polynomials that can be classified as generalized Faber polynomials are exactly those given in Theorem 1 and there are no others.

Theorem 5. The only Jacobi polynomials that can be classified as generalized Faber polynomials are exactly those given in Theorem 1 and there are no others. Proof. From Theorem 2 we need only to consider the Jacobi polynomials which can be classified as generalized Faber polynomials of the elliptic region $\mathscr{A}(c)$. Without loss of generality, we may take $c=1$. For $\psi(t)=\frac{1}{2}(t+1 / t)$, the differential equation (3.26) now takes the form

$$
\begin{aligned}
\left(1-u^{2}\right) F^{\prime \prime}(u) & +[(\beta-\alpha) \psi(t)-B(t) \psi(t) u] F^{\prime}(u) \\
& +C(t) \psi^{2}(t) F(u)=0
\end{aligned}
$$

where

$$
\psi(t) B(t)=\frac{q}{t^{2}}+(q+4)+\frac{1}{2} t\left(t^{2}-\frac{1}{t^{2}}\right)\left(\frac{R^{\prime}(t)}{R(t)}\right)
$$

and

$$
\begin{aligned}
& C(t) \psi^{2}(t)=\frac{1}{4}\left(t^{2}+1\right)^{2}\left\{\frac{\left.-8(q+1) t^{2}-(1-q)\right]\left[t^{2}-1\right]}{\left(t^{4}-1\right)^{2}}\right. \\
&+(1-2 q) \frac{1}{t}\left(\frac{R^{\prime}(t)}{R(t)}\right)+\frac{8 t}{\left(t^{4}-1\right)}\left(\frac{R^{\prime}(t)}{R(t)}\right) \\
&\left.+\left(\frac{R^{\prime}(t)}{R(t)}\right)^{2}+\frac{d}{d t}\left(\frac{R^{\prime}(t)}{R(t)}\right)\right\} .
\end{aligned}
$$

Case (i). $C(t)=0$. From (3.30), one obtains

$$
(1-2 q) G^{\prime}(t)+t G^{\prime \prime}(t)=0
$$

which upon solving gives

$$
G(t)=\left[\begin{array}{ll}
c_{0} t^{2 q}+c_{1} & \text { if } q \neq 0 \\
c_{0} \ln t+c_{1} & \text { if } q=0
\end{array}\right.
$$

where $c_{0}$ and $c_{1}$ are constants. In view of (3.19) and (3.36), one has

$$
R(t)=\left[\begin{array}{ll}
\frac{\left(t^{2}+1\right)}{\left(t^{2}-1\right)} c_{0}, & \\
\frac{\left(t^{2}+1\right)\left(c_{0} t^{2 q}+c_{1}\right)}{\left(t^{2}-1\right)} & \text { if } q \neq 0, \\
\frac{\left(t^{2}+1\right)\left(c_{0} \ln t+c_{1}\right)}{\left(t^{2}-1\right)} & \text { if } q=0 .
\end{array}\right.
$$


Equation (3.33) now gives

$$
\frac{F^{\prime \prime}(u)}{F^{\prime}(u)}=-\frac{[(\beta-\alpha) \psi(t)-B(t) \psi(t) u]}{\left(1-u^{2}\right)} .
$$

For $F(u)$ to be independent of $t, \beta$ must be equal to $\alpha$ and

$$
B(t) \psi(t)=a \text { (constant). }
$$

By combining (3.34) and (3.39) one obtains, after solving the resulting differential equation in $R(t)$,

$$
R(t)=c_{2} \frac{t^{2 q}\left(t^{2}-1\right)^{(a-2 q-4) / 2}}{\left(t^{2}+1\right)^{(a-4) / 2}}
$$

for some constant $c_{2}$. By comparing (3.37) and (3.40), one deduces that the only possible solution is

$$
R(t)=c_{0} \frac{\left(t^{2}+1\right)}{\left(t^{2}-1\right)}
$$

when $a=2, q=0$. Equation (3.38) now becomes

$$
\frac{F^{\prime \prime}(u)}{F^{\prime}(u)}=\frac{2 u}{1-u^{2}}
$$

which gives

$$
F(u)=\frac{1}{2} \ln \left\{\frac{1+u}{1-u}\right\} .
$$

Although $R(t)$ and $F(u)$ have been calculated explicitly, no generalized Faber polynomials exist in this case since $R(t), F(u)$ are the same as those given in (3.17a) and (3.17b).

Case (ii). $C(t) \neq 0$ and $\beta \neq \alpha$. By taking the partial derivative of (3.33) with respect to $t$, we obtain

$$
\frac{F^{\prime}(u)}{F(u)}=-\frac{\frac{\partial}{\partial t}\left(C(t) \psi^{2}(t)\right)}{(\beta-\alpha) \frac{\partial \psi(t)}{\partial t}-\left(\frac{\partial}{\partial t} B(t) \psi(t)\right) u}
$$

For $F(u)$ to be independent of $t$, we must have

$$
\frac{\partial}{\partial t}(B(t) \psi(t))=\eta \frac{\partial \psi(t)}{\partial t} \text { or } \quad B(t) \psi(t)=\eta \psi(t)+a
$$

and

$$
\frac{\partial}{\partial t}\left(C(t) \psi^{2}(t)\right)=\frac{d}{2} \frac{\partial \psi(t)}{\partial t} \quad \text { or } \quad C(t) \psi^{2}(t)=\frac{d}{2} \psi(t)+\frac{e}{4}
$$

for some constants $\eta, a, d$, and $e$. By combining (33.4), (3.45) for $\psi(t)=$ $\frac{1}{2}(t+1 / t)$ and solving for $R(t)$, we have

$$
R(t)=c_{0} \frac{t^{2 q}\left(t^{2}-1\right)^{(a-2 q-4) / 2}(t-1)^{\eta / 2}}{\left(t^{2}+1\right)^{(a-4) / 2}(t+1)^{\eta / 2}} .
$$


In view of (3.35) and (3.46), we have

$$
\begin{aligned}
& \frac{1}{4}\left(t^{2}+1\right)^{2}\left\{\frac{-8\left[(q+1) t^{4}-2 t^{2}+(1-q)\right]}{\left(t^{4}-1\right)^{2}}\right. \\
& \left.+(1-2 q) \frac{1}{t} y+\frac{8 t}{\left(t^{4}-1\right)} y+y^{2}+y^{\prime}\right\}=\frac{d}{4}\left(t+\frac{1}{t}\right)+\frac{e}{4}
\end{aligned}
$$

where $y=\frac{R^{\prime}(t)}{R(t)}$. Taking the logarithmic derivative of (3.47) yields

$$
y=\frac{\eta t^{3}+2(a-q-4) t^{2}+\eta t-2 q}{t\left(t^{4}-1\right)},
$$

which, upon substituting in (3.48) and simplifying, gives

$$
\begin{aligned}
(3.50)[\eta & +2 q \eta+d]\left[t^{6}+1\right]+\left[-4 q^{2}+(4 a-12) q-\eta^{2}+e+4 a-8\right]\left[t^{5}+t\right] \\
& +[6 \eta q+(11-4 a) \eta-d]\left[t^{4}+t^{2}\right] \\
& +\left[-8 q^{2}+(8 a-24) q-2 \eta^{2}-2 e-4 a^{2}+16 a-16\right] t^{3}=0 .
\end{aligned}
$$

Therefore,

$$
\begin{aligned}
& \eta+2 q \eta+d=0 \\
& \quad-4 q^{2}+(4 a-12) q-\eta^{2}+e+4 a-8=0 \\
& -4 q^{2}+(4 a-12) q-\eta^{2}-e-2 a^{2}+8 a-8=0 \\
& 6 \eta q+(11-4 a) \eta-d=0
\end{aligned}
$$

solving these equations gives the following solutions:

$\eta=0, \pm 1, d=-\eta(1+2 q), e=-a(a-2)$ and $a=2 q+3$ unless $\eta=0$.

First, let us assume that $\eta \neq 0$. Then,

(a) if $\eta=1, d=-(1+2 q), a=2 q+3, e=-(2 q+3)(2 q+1)$ and

(b) if $\eta=-1, d=(1+2 q), a=2 q+3, e=-(2 q+3)(2 q+1)$.

Equation (3.44) can now be written in the form

$$
\frac{F^{\prime}(u)}{F(u)}=-\frac{d}{2 \eta} \frac{1}{(\beta-\alpha) / \eta-u} \text {. }
$$

Thus,

$$
F(u)=[(\beta-\alpha) / \eta-u]^{d / 2 \eta} .
$$

Since $F(u)$ has a singularity at $u=1$, then $\eta=\beta-\alpha$. Therefore, in case (a), $\beta=\alpha+1,2 q=2 \alpha+2$, and from (3.47), (3.53) we obtain

$$
\begin{aligned}
& R(t)=c_{0} \frac{t^{2 a+2}}{\left(t^{2}+1\right)^{\alpha+1 / 2}(t+1)} \quad(\text { cf. }(3.12 \mathrm{a})), \\
& F(u)=[1-u]^{-(\alpha+3 / 2)} \quad(\mathrm{cf} .(3.12 \mathrm{~b})) .
\end{aligned}
$$


Thus, we have a generating function for generalized Faber polynomials of the form

$$
c_{0} \frac{(t-1) t^{2 \alpha+2}}{\left(t^{2}+1\right)^{(\alpha+3 / 2)}}\left[1-\frac{x}{\frac{1}{2}(t+1 / t)}\right]^{-(\alpha+3 / 2)} \quad(\text { cf. (3.11)). }
$$

From Theorem 1(d), we conclude that the generalized Faber polynomials in this case are

$$
\left\{\frac{(2 \alpha+2)_{n}}{(\alpha+1)_{n}} P_{n}^{(\alpha, \alpha+1)}(x)\right\}_{n=0}^{\infty} .
$$

In case (b), $\alpha=\beta+1,2 q=2 \beta+2$, and (3.47), (3.53) now give

$$
\begin{aligned}
& R(t)=c_{0} \frac{t^{2 \beta+2}}{\left(t^{2}+1\right)^{\beta+1 / 2}(t-1)} \quad(\mathrm{cf} .(3.15 \mathrm{a})), \\
& F(u)=[1-u]^{-(\beta+3 / 2)} \quad(\mathrm{cf} .(3.15 \mathrm{~b})) .
\end{aligned}
$$

Thus, we have a generating function for generalized Faber polynomials of the form

$$
c_{0} \frac{t^{2 \beta+2}(t+1)}{\left(t^{2}+1\right)^{\beta+3 / 2}}\left[1-\frac{x}{\frac{1}{2}(t+1 / t)}\right]^{-(\beta+3 / 2)} \quad \text { (cf. (3.14)). }
$$

Again, from Theorem 1(c), we conclude that the generalized Faber polynomials in this case are

$$
\left\{\frac{(2 \beta+2)_{n}}{(\beta+1)_{n}} P_{n}^{(\beta+1, \beta)}(x)\right\}_{n=0}^{\infty} .
$$

Now we consider the case $\eta=0$. In this case (3.44) becomes

$$
\frac{F^{\prime}(u)}{F(u)}=-\frac{\frac{\partial}{\partial t}\left(C(t) \psi^{2}(t)\right)}{(\beta-\alpha) \partial \psi(t) / \partial t}
$$

and for $F(u)$ to be independent of $t$ and have a singularity at $u=1$, $\frac{\partial}{\partial t}\left(C(t) \psi^{2}(t)\right)$ must be zero and $\beta$ must be equal to $\alpha$ with contradicts our assumption.

Case (iii). If $C(t) \psi^{2}(t) \neq 0$ and $\beta=\alpha$. In this case equation (3.44) becomes

$$
\frac{F^{\prime}(u)}{F(u)}=\frac{\frac{\partial}{\partial t}\left(C(t) \psi^{2}(t)\right)}{\frac{\partial}{\partial t}(B(t) \psi(t)) u}
$$

and for $F(u)$ to be independent of $t$ and have a singularity at $u=1$, we must have

$$
B(t) \psi(t)=a \quad \text { and } \quad C(t) \psi^{2}(t)=e / 4 .
$$

To solve these two equations simultaneously, we note that they are equivalent to (3.45), (3.46) with $\eta=0$ and $d=0$ respectively. Therefore, it is easy to 
see that in this case we obtain a system of equations similar to (3.51) but with $\eta=0=d$. Upon solving this new system of equations, we have

$$
e=-a(a-2), \quad a=\left\{\begin{array}{l}
2(q+1), \\
2(q+2),
\end{array}\right.
$$

and hence by (3.47)

$$
R(t)=\left\{\begin{array}{l}
c_{0} \frac{t^{2 q}}{\left(t^{2}+1\right)^{(q-1)}\left(t^{2}-1\right)} \quad \text { if } a=2(q+1) \quad(\mathrm{cf} .(3.6 \mathrm{a})), \\
c_{0} \frac{t^{2 q}}{\left(t^{2}+1\right)^{q}} \quad \text { if } a=2(q+2) \quad(\mathrm{cf} .(3.9 \mathrm{a}))
\end{array}\right.
$$

Equation (3.33) now becomes

$$
\left(1-u^{2}\right) F^{\prime \prime}(u)-2(q+1) u F^{\prime}(u)-q(q+1) F(u)=0, \quad \text { if } a=2(q+1)
$$

and

$$
\left(1-u^{2}\right) F^{\prime \prime}(u)-2(q+2) u F^{\prime}(u)-(q+1)(q+2) F(u)=0, \quad \text { if } a=2(q+2) .
$$

Thus,

$$
F(u)=\left\{\begin{array}{l}
(1-u)^{-q} \quad \text { if } a=2(q+1) \quad(\mathrm{cf} .(3.6 \mathrm{~b})), \\
(1-u)^{-(q+1)} \quad \text { if } a=2(q+2) \quad(\mathrm{cf} .(3.9 \mathrm{~b})),
\end{array}\right.
$$

and consequently there are two generating functions for two sets of generalized Faber polynomials

$$
\frac{t^{2 q}}{\left(t^{2}+1\right)^{q}}\left[1-\frac{x}{\frac{1}{2}(t+1 / t)}\right]^{-q} \quad \text { if } a=2(q+1) \quad(\mathrm{cf} . \quad(3.5))
$$

and

$$
\frac{\left(t^{2}-1\right) t^{2 q}}{\left(t^{2}+1\right)^{q+1}}\left[1-\frac{x}{\frac{1}{2}(t+1 / t)}\right]^{-(q+1)} \text { if } a=2(q+2) \quad(\mathrm{cf} . \quad(3.8)) .
$$

Since $2 q=\alpha+\beta+1=2 \alpha+1$ and for the Gegenbauer polynomials $\alpha=\lambda-\frac{1}{2}$, hence $q=\lambda$, we conclude from Theorem 1-b, $\mathrm{c}$ that the generalized Faber polynomials in this case are

$$
\left\{C_{n}^{\lambda}(x)\right\}_{n=0}^{\infty} \quad \text { if } a=2(q+1) \text { and } \quad\left\{\frac{(n+\lambda)}{\lambda} C_{n}^{\lambda}(x)\right\}_{n=0}^{\infty} \quad \text { if } a=2(q+2) \text {. }
$$

Q.E.D.

Finally, let us remark that the generalized Faber polynomials have also been defined for unbounded and multiply-connected domains as well [7, p. 145].

Although some normalized Hermite polynomials are limits of generalized Faber polynomials, since [12, p. 197]

$$
\frac{H_{n}(x)}{n !}=\lim _{\lambda \rightarrow \infty}\left[\lambda^{-n / 2} C_{n}^{\lambda}\left(\lambda^{-1 / 2} x\right)\right],
$$

they are not themselves generalized Faber polynomials for any region, no matter how we normalize them. 
Corollary 1. For any normalization whatsoever, the Laguerre and Hermite polynomials are not generalized Faber polynomials for any region $\mathbf{B}$.

Proof. In the cases where $\mathbf{B}$ is simply connected, whether bounded or not, one uses the same arguments given in Theorems 2, 3 together with the Laguerre and Hermite differential equations to show that there exists no function $F(u)$ for which (1.5) holds. The case where $\mathbf{B}$ is multiply-connected can be considered as a combination of the above two.

Acknowledgment. This research was done while the author was spending part of his sabbatical leave at the Mathematics and Computer Centre (Stichting Mathematisch Centrum) in Amsterdam and he would like to take this opportunity to thank Professor M. Hazewinkel, the head of the Mathematics Department, for his hospitality and Professor T. Koornwinder for his interest in this research and for making constructive comments.

\section{REFERENCES}

1. R. Askey, Orthogonal polynomials and special functions, Regional Conference Series in Appl. Math., vol. 21, SIAM, 1975.

2. G. Faber, Über polynomische Entwicklungen, Math. Ann. 57 (1903), 389-408; 64 (1907), 116-135.

3. Ya. L. Geronimus, Polynomials, orthogonal on a a circle and on an interval, Fizmatgiz, Moscow, 1958.

4. R. Gilbert, Integral operator methods in bi-axially symmetric potential theory, Contrib. Differential Equations 2 (1963), 441-456.

5. B__ Bergman's integral operator method in generalized axially symmetric potential theory, J. Math. Phys. 5 (1964), 983-997.

6. Z. Nehari, On the singularities of Legendre expansions, Indiana Math. J. 5 (1956), 987-992.

7. V. J. Smirnov and N. Lebedev, Functions of a complex variable, M. I. T. Press, Cambridge, Mass., 1968.

8. H. Srivatstave and H. Manocha, A treatise on generating functions, Ellis Horwood, West Sussex, England, 1984.

9. P. Suetin, The basic properties of Faber polynomials, Uspekhi Mat. Nauk 19 (1964), No. 4, 125-154; Russian Math. Surveys 19 (1964), No. 4, 121-149.

10. __ Fundamental properties of polynomials orthogonal on a contour, Usepkhi Mat. Nauk 21 (1966), No. 2 (128), 41-88; Russian Math. Surveys 21 (1966), No. 2, 35-83.

11. __ Polynomials orthogonal over a region and Bieberbach polynomials, Proc. Steklov Inst. Math., No. 100 (1971); English transl., Amer. Math. Soc., Providence, R. I., 1974.

12. G. Szegö, Orthogonal polynomials, Amer. Math. Soc. Colloq. Publ., vol. 23, Amer. Math. Soc., Providence, R. I., 1975.

13. A. Zayed, M. Freund and E. Görlich, A theorem of Nehari revisted, Complex Variables Theory Appl. 10 (1988), 11-22.

Department of Mathematics, California Polytechnic State University, San Luis ObisPo, CALIFORNIA 93407 